\title{
On Finite-Gap Elliptic Solutions of the KdV Equation
}

\author{
A.M. KOROSTIL' \\ Institute of Metal Physics, 252680, Kyïv-142, Vernadsky str., 36, Ukraïna
}

\begin{abstract}
We present a simple and general method for calculation of finite-gap elliptic solutions of the KdV equation as an isospectral deformation of Schrödinger potential based on their representation by rational functions of the elliptic Weierstrass functions.
\end{abstract}

\section{Introduction}

Solutions of algebraically integrable nonlinear equations in partial derivatives on $x$ and $t$, admitting the Lax representation $\mathrm{L}_{\mathrm{t}}=[\mathrm{A}, \mathrm{L}][1]$, which are solutions of the spectral and isospectral deformation problems for the linear differential L-operator, are expressed over $n$-dimensional theta functions containing implicit parameters, a calculating of which is a special problem [1-4].

In this paper, a simple and general method for calculation of finite-gap elliptic solutions of the KdV equation as an isospectral deformation of the Schrödinger potential based on their representation by rational functions of the elliptic Weierstrass functions [5] and on employment of trace formulae is proposed that reduces to the problem of solving an unified algebraic system of equations. It is shown that substitution of these rational functions into the trace formulae and the Lax dynamic equations yields, under the condition of vanishing the principal part in poles, the algebraic equations, the compatibility conditions of which lead to the elliptic solutions.

\section{Equations for the Schrödinger potential}

The finite-gap solution $U(x, t)$ of the $\mathrm{KdV}$ equation is the potential associated with the Lax representation of one-dimensional Schrödinger operators $H=-d^{2} / d x^{2}+U(x, t)$. Finite-gap eigenfunctions satisfying the equation $H \Psi(x, t, E)=E \Psi(x, t, E))$ have the form $\Psi(x, t, E)=\sqrt{\chi_{R}(x, t, E)} \exp \left(\int^{x} d x \chi_{R}(x, t, E)\right)$, where $\chi_{R}$ is a real function that can be represented in the form of the asymptotic series

$$
\chi_{R}(x, E)=\sqrt{E}\left(1+\sum_{n=0}^{\infty} \frac{(-1)^{n}}{2^{2 n+1}} \chi_{2 n+1}(x) E^{-(n+1)}\right)
$$

(further for simplicity we shall omit arguments of the functions $\chi_{n}$ ). The coefficients of (1) satisfy the well-known [1] recurrent relation

Copyright (c) 1997 by Mathematical Ukraina Publisher. All rights of reproduction in any form reserved. 


$$
\chi_{n+1}=\frac{d}{d x} \chi_{n}+\sum_{k=1}^{n-1} \chi_{k} \chi_{n-k}, \quad \chi_{1}=-U(x) .
$$

In the case of $g$-gap spectra (which have $g$ gaps and $2 g+1$ boundaries $\left\{E_{i}\right\}$ ), the real function $\chi_{R}$ is described by the expression

$$
\chi_{R}(x, E)=\frac{\sqrt{P(E)}}{\prod_{n=1}^{g}\left(E-\mu_{n}(x)\right)}, \quad P(E)=\prod_{n=1}^{2 g+1}\left(E-E_{n}\right),
$$

which after removal of parentheses transforms to the form

$$
\chi_{R}(x, E)=\frac{\sqrt{\sum_{n=0}^{2 g+1} a_{n} E^{-n}}}{\sum_{n=0}^{g} b_{n} E^{-n}}, \quad a_{0}=1, \quad b_{0}=1 .
$$

Here $a_{n}$ and $b_{n}$ are symmetrized products of spectral boundaries $E_{j}$ and $\mu$-functions of the $n$th order, respectively. The expression (4) can be represented similarly to the case (1) in the form of the asymptotic series

$$
\chi_{R} \sim \sqrt{E}\left(1+\sum_{n=1}^{\infty} A_{n} E^{-n}\right),
$$

Equating coefficients at equal powers of the independent variable $E^{-1}$ in the expressions (1) and (5), we get trace formulae

$$
A_{n+1}=\frac{(-1)^{n}}{2^{2 n+1}} \chi_{2 n+1},
$$

which are relations between symmetrized products $b_{n}, a_{n}$ and polynomials of the $n$-th order in the potential $U(x, t)$ with its derivatives. A substitution into the system of equations (6) of the general expression for the elliptic potential

$$
U(z)=\left\{\sum_{n=1}^{N} \alpha_{n} \wp^{n}(z)+\sum_{i} \sum_{n_{i}=1}^{M_{i}} \alpha_{n_{i}}\left(\wp(z)-h_{i}\right)^{-M-n_{i}}+C_{i}\right\}+\wp^{(1)} \tilde{R}(\wp),
$$

$\left(C_{i}\right.$ is a constant, $\wp$ is a Weierstrass function, $\tilde{R}(\wp)$ is a rational function of $\left.\wp\right)$ leads to equations both for potential parameters and for symmetrized products of boundaries (which are coefficients of the spectral polynomial $P(E)$ ).

1. One-gap initial potentials, $g=1$. According to the equation (6), in this case $b_{1} \neq 0$ and $b_{n}=0$ when $n \geq 2$. Taking into account the explicit expressions for $h_{i}, A_{i}, b_{1}$, we will obtain the system of equations

$$
\begin{aligned}
b_{2}= & \frac{1}{8}\left(3 U^{2}-U^{(2)}\right)+\frac{1}{4} a_{1} U+\frac{1}{2} a_{2}-\frac{1}{8} a_{1}^{2}=0 \\
b_{3}= & -\frac{1}{32}\left(U^{(4)}+10 U^{3}-5 U^{(1)^{2}}-10 U U^{(2)}\right)-\frac{1}{16} a_{1}\left(3 U^{2}-U^{(2)}\right)+ \\
& \frac{1}{16} U\left(a_{1}^{2}-4 a_{2}\right)+\frac{1}{2} a_{3}+\frac{1}{4} a_{1} a_{2}-\frac{1}{16} a_{1}^{3}=0 .
\end{aligned}
$$


Substituting the general expression (7) into the equation (8) and equating to zero principal parts at poles $\wp=h, \wp=e_{i}$ and at pole $y=\infty$, where $y=1 / \wp$, we obtain the system of equalities, compatibility conditions of which give all possible initial 1-gap elliptic potentials in the form

$$
\begin{aligned}
& \text { 1) } U(z)=2 \wp(z), P(E)=E^{3}-\frac{1}{4} g_{2} E-\frac{1}{4} g_{3}, E_{i}=e_{i} \quad(i=1,2,3), \\
& \text { 2) } U(z)=2 \wp(z)+2\left(\wp\left(z-\varphi^{(1)}\right)+\wp\left(z+\varphi^{(1)}\right)\right)-4 \wp\left(\varphi^{(1)}\right),
\end{aligned}
$$

(here $z=i x+\omega$ means a complex variable). The potentials 1 and 2 coincide with the known Lamé potential [5] and the potential obtained by Smirnov in [4] by an algebraic geometrical method.

2. Two-gap initial potentials, $g=2$. In this case the elliptic potentials and corresponding spectra are described by the system of equations (6) in which $\left.b_{i}\right|_{i \geq 3}=0$ and $\left.b_{i}\right|_{i \leq 2} \neq 0$. The first of these equations $(n=3)$ which is equivalent to the equation (9) determines the potential parameters and the coefficients $a_{i},(i=1, \ldots, 3)$ of the spectral polynomial $P(E)$. Other two equations corresponding to $n=4,5$ determine the coefficients $a_{4}, a_{5}$. Substituting the general elliptic potential (7) in the equation (9) for $b_{3}=0$ and equating to zero principal parts at poles $\wp=h, \wp=e_{i}$ and pole $y=\infty$, where $y=1 / \wp$, we obtain the system of the equalities, the compatibility conditions of which lead to the expressions

$$
\begin{aligned}
\text { 1) } U(z) & =6 \wp(z), P(E)=E^{5}-\frac{21}{4} g_{2} E^{3}-\frac{27}{4} g_{3} E^{2}+\frac{27}{4} g_{2}^{2} E-\frac{81}{4} g_{2} g_{3}, \\
\text { 2) } U(z) & =6 \wp(z)+2 \wp\left(z+\omega_{i}\right)-2 e_{i}, \\
\text { 3) } U(z) & =6 \wp(z)+2 \wp\left(z+\omega_{i}\right)+2 \wp\left(z+\omega_{k}\right)+2 e_{k}, \\
\text { 4) } U(z) & \left.\left.=6 \wp(z)+2 \wp\left(z-\varphi^{(2)}\right)+2 \wp\left(z+\varphi^{(2)}\right)\right)-4 \wp\left(\varphi^{(2)}\right)\right),
\end{aligned}
$$

(where we omit the known expressions for a spectral polynomial and the equation for $\varphi^{(2)}$ ) exhausting all initial 2-gap elliptic potentials.

The potentials 1 and 2, 3 are the known Lamé and Treibich-Verdier potentials [6], respectively, and the potential 4 coincides with the potential obtained by Smirnov [4]

Initial three-gap elliptic potentials. In this case elliptic potentials and spectral polynomials are determined by the system (6) in which $b_{i \geq 4}=0$. Substituting (7) in (6) and equating the principal parts at poles $\wp=h, \wp=e_{i}$ to zero, we get all possible initial 3-gap elliptic potentials including the potentials and the spectral polynomials of the form

$$
\begin{aligned}
\text { 1) } U(z)= & 12 \wp(z), P(E)=E^{7}-\frac{63}{2} g_{2} E^{5}-\frac{297}{2} g_{3} E^{4}+\frac{4185}{16} g_{2}^{2} E^{3}- \\
& \frac{18225}{8} g_{2} g_{3} E^{2}+\left(\frac{91125}{16} g_{3}^{2}-\frac{3375}{16} g_{2}^{3}+\right) E, \\
\text { 2) } U(z)= & 12 \wp(z)+12\left(\wp \left(z+\varphi^{(3)}+\wp\left(z-\varphi^{(3)}\right) .\right.\right.
\end{aligned}
$$

Here 1 notes the known 3-gap elliptic Lamé potential and the corresponding spectral polynomial and 2 notes elliptic potential obtained by Smirnov [4]. Possible 3-gap potentials are expressed only as a linear combination of the Lamé potentials without and with shifts of arguments on half-periods $\omega_{i}$ or with a shift on the value $\varphi^{(3)}$ depending on spectral parameters $\tau, \omega$. 


\section{Dynamic of finite-gap elliptic solutions}

The time evolution of the eigenfunction of the Schrödinger operator $\mathrm{H}$ is described by the equation

$$
\frac{\partial}{\partial t} \Psi(x, t, E)=A \Psi(x, t, E) A=4 \frac{\partial^{3}}{\partial x^{3}}-3\left(U \frac{\partial}{\partial x}+\frac{\partial}{\partial x} U\right)
$$

whence in view of its $\chi_{R}$-representation we can obtain the equation

$$
\frac{\partial}{\partial t} \chi_{R}(x, t, e)=\frac{\partial}{\partial x}\left(\lambda \chi_{R}(x, t, E)\right), \lambda=-2(U(x, t, E)+E) .
$$

Substituting here the explicit expression for the $\chi_{R}$-function (4) we get the relation

$$
\sum_{n=0}^{N} \dot{b}_{n} E^{N-n}=2 \sum_{n=0}^{N}\left(U b_{n}\right)^{\prime}+2 b_{n}^{\prime} E^{N+1-n},
$$

Equating the coefficients at equal powers of $E$ of the left- and right-hand parts of this equality, we get the dynamic equation

$$
\frac{\partial}{\partial t} b_{n}=2 \frac{\partial}{\partial x} b_{n+1}+4 \frac{\partial}{\partial x}\left(U b_{n}\right), \quad n=(1, \ldots, g)
$$

which at $n=2$ coincides with the KdV equation.

Thus in the general case of a time dependence, the finite-gap elliptic solutions must satisfy both the trace formulae (6) and the dynamic equation (11). Substituting the expression (7) in these system yields, under the condition of vanishing the principal parts at poles, the equalities, the compatibility conditions of which bring up to the elliptic potential

$$
U(x, t)=2 \sum_{i=1}^{N} \wp\left(z-x_{j}(t)\right),
$$

which in the 2-gap case satisfy the equations

$$
\frac{\partial}{\partial t} x_{j}(t)=4 X_{i}(t)
$$

where

$$
X_{i}(t)=-3 \sum_{j \neq i} \wp\left(x_{i}(x, t)-x_{j}(x, t)\right)
$$

and

$$
\sum_{j \neq i} \wp^{\prime}\left(x_{i}(t)-x_{j}(t)\right)=0
$$

with the initial conditions that are described by the above-mentioned initial elliptic potentials.

The symmetrized products of the $X_{i}$-functions for arbitrary elliptic solutions are determined from the dynamic equations and trace formulae under the compatibility conditions, if we substitute (12) into (6), (11) and the functions $X_{i}(t)$ can be presented as roots of 
the corresponding polynomial. In the considered 2-gap case for the different initial elliptic potentials $1,2,3$, we get, respectively, the equations

$$
\begin{array}{r}
4 X^{3}-g_{2} X+9 g_{3}+\frac{16}{9} \wp(\tilde{8} i t)=0, \\
9\left(X-E_{2}\right)\left(X-E_{3}\right)\left(X+4 e_{i}-e_{k}\right)^{2}+4\left(X+5 e_{i}\right)\left(\tilde{\wp}(8 i t)-\tilde{e_{j}}\right)=0, \\
9 P_{5}(X)+4\left(X-3 e_{i}-9 e_{j}\right)\left(X-3 e_{i}-9 e_{k}\right) \tilde{\wp}(8 i t)=0,
\end{array}
$$

where $\tilde{\wp}(z)$ is determined by the relation $z=\int_{0}^{\tilde{\wp}} d x /\left(\sqrt{4 x^{3}-\tilde{g}_{2} x-\tilde{g}_{3}}\right),\left(\tilde{g}_{i}\right.$ and $\tilde{e}_{i}$ are expressed via the elliptic invariants $g_{2}, g_{3}$ and $\left.e_{i}=\wp\left(\omega_{i}\right)\right), P(X)$ is the fifth-order polynomial presented in [3]. These equations permit to solve the evolution equation (11). Solutions which coincide with results of Enol'skii and Eilbeck [3] are based on employment of the inverse scattering and reduction method.

Similarly our consideration can be continued for calculating finite-gap elliptic solutions of larger order $g>2$.

The author is grateful to E.D.Belokolos, V.Z.Enol'skii for valuable discussions.

This work is supported by the International Science Foundation through Grant No. UB6200.

\section{References}

[1] Zakharov V.E., Manakov S.V., Novikov S.P., Pitaevskii L.P., Theory of Soliton. The Inverse Scattering Method, Nauka, Moscow, 1980.

[2] Belokolos E.D., Bobenko A.I., Eno'lskii V.Z., Its A.R., Matveev V.B., Algebraic Geometrical Methods in Theory of Integrable Equations, Springer, Berlin, 1994.

[3] Enol'skii V.Z., Eilbeck J.C., On the Two-Gap Locus for the Calogero-Moser Model, J. Phys. A, 1995, V.28, N 3, 1069-1087.

[4] Smirnov A.O., Finite-Gap Elliptic Solutions of the KdV Equation, Acta Mathematicae Applicandae, 1994, V.36, N 3, 31-55.

[5] Bateman E.G., Erdeyi A., Higher Transcendental Functions. Elliptic and Automorphic Functions. III, McGraw-Hill, New-York, 1955.

[6] Treibich A., Verdier J.L., New Elliptic Solitons, Preprint, Paris, 1987. 